\title{
First statistics of the turbulence outer scale at Dome C
}

\author{
A. Ziad, E. Aristidi, A. Agabi, J. Borgnino, F. Martin, and E. Fossat \\ UMR 6525 H. Fizeau, Université de Nice Sophia-Antipolis, CNRS, Observatoire de la Côte d'Azur, Parc Valrose, 06108 Nice, France \\ e-mail: ziad@unice.fr
}

Received 12 March 2008 / Accepted 3 July 2008

\section{ABSTRACT}

\begin{abstract}
Context. The outer scale of wavefronts is of interest for the dimensioning and the optimisation of the High Angular Resolution techniques such as Interferometry and Adaptive Optics, particularly for the new generation of telescopes such as the Extremely Large Telescopes (ELT).

Aims. We aim to provide the first statistics of the outer scale at Dome $\mathrm{C}$ site in Antarctica to finalize the characterization of this site. Methods. A new version of the Generalized Seeing Monitor has been developed for extremely cold conditions. Two DIMMs (Differential Image Motion Monitor) were coupled to extract Angle-of-Arrival (AA) fluctuations using CCD detectors. Correlations of these AA fluctuations for different baselines lead to outer scale estimations.

Results. For the first time, statistics of the outer scale at Dome $\mathrm{C}$ are provided leading to small values in the surface layer at Dome $\mathrm{C}$. These small outer scale values compared to temperate sites considerably reduce the fringe excursion of interferometers and the low orders of the normalized amplitude of atmospheric Zernike coefficients, particularly the tip-tilt.

Conclusions. The Dome $\mathrm{C}$ small outer scale combined with the large coherence time and large isoplanatic angle are very useful for the development of Adaptive Optics systems and long-baseline interferometers.
\end{abstract}

Key words. instrumentation: interferometers - instrumentation: adaptive optics - atmospheric effects - site testing

\section{Introduction}

The wavefront outer scale is a relevant parameter for the experimental performance evaluation of large aperture telescopes. The actual size of the outer scale has long been controversial, with measured values ranging from less than $10 \mathrm{~m}$ to more than $2 \mathrm{~km}$. What is not controversial is the conclusion that when the diameter of the telescope approaches or exceeds the size of the outer scale, the optical consequences of atmospheric turbulence are dramatically changed from their traditional Kolmogorov behavior. In particular, power in the lowest Zernike aberration modes, e.g., tip and tilt and the overall stroke required for an adaptiveoptics system can be much reduced (Winkler 1991). A finite outer scale has implications for interferometry as well (Conan 2000).

In the context of the current interest for the design of groundbased astronomical facilities at Dome $\mathrm{C}$ in Antarctica, a reliable estimation of the outer scale has become even more important. A new version of the Generalized Seeing Monitor (GSM) dedicated to extremely cold conditions has been developed and installed at Dome $\mathrm{C}$ for the completion of its characterization. This version consists of coupling two DIMMs ("Differential Image Motion Monitor") to extract Angle-of-Arrival (AA) fluctuations using CCD detectors. This new GSM version is described in Sect. 2 as well as the site instrumentation set-up. A statistical study of outer scale measurements obtained at Dome C in autumn-winter 2006 is presented in Sect. 3. Implications for High Angular Resolution (HAR) techniques are discussed in Sect. 4.

\section{Dome C GSM version and instrument set-up}

The GSM version installed at Dome $\mathrm{C}$ is based on two identical DIMMs simultaneously observing the same star (Fig. 1).
Each DIMM is a telescope equipped with a mask having subapertures of $6 \mathrm{~cm}$ diameter separated by $20 \mathrm{~cm}$. This mask is placed at the entrance of the telescope. One of the sub-apertures is equipped with a small angle prism (deviation 30 arcsec), the other one with a glass parallel plate. We use a SchmidtCassegrain Celestron C11 telescope (diameter $280 \mathrm{~mm}$ ) with a 2xBarlow lens (this gives an equivalent focal length of $5600 \mathrm{~mm}$ ). A digital CCD camera (PCO PixelFly) is placed in a temperature controlled box (temperature around $-20{ }^{\circ} \mathrm{C}$ ) which is installed at the focus of the telescope. The exposure time is less than $5 \mathrm{~ms}$ and the pixel size is $9.9 \mu \mathrm{m}$ corresponding to $0.75^{\prime \prime}$. The CCD camera is operated in the visible in the spectral range from $290 \mathrm{~nm}$ to $1100 \mathrm{~nm}$ with maximum sensitivity at $500 \mathrm{~nm}$. Software has been developed to perform real time data processing. Each short exposure frame is thresholded to eliminate the background, then the two stellar images are easily detected in two small $20 \times 20$ pixels windows and their photocenter coordinates computed by means of a simple barycenter formula (Aristidi et al. 2005). Each telescope is set on an equatorial mount (Astro-Physics 900). The mounts are installed on 2 massive wooden pillars. The foundations of these pillars are independent in order to avoid correlated vibrations between the 2 DIMMs. The top of each DIMM is at $3.5 \mathrm{~m}$ above the ground level. This situation is not optimal due to the ground layer turbulence contribution but allows us to avoid instrument vibrations that prevail if installed higher on a wood platform (Aristidi et al. 2005, Fig. 3). This equipment has been customized to work in Antarctic cold conditions.

At the focus of each GSM telescope are two images of the same star recorded with the CCD camera. The two images are moving due to the turbulence and the motion of their barycenters is recorded in the $X$ and $Y$ directions. We obtain 8 curves (4 per telescope) of these barycenter motions. Every minute a set of 6 cross-correlations of the $X$-direction motion (less 


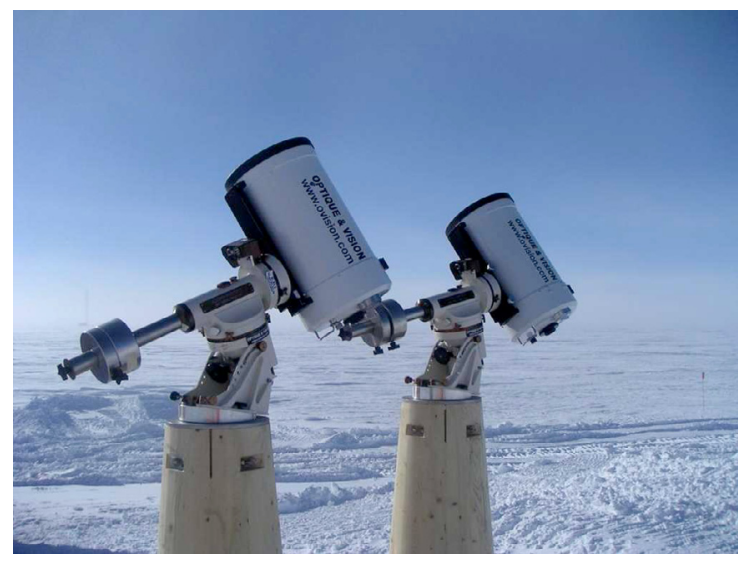

Fig. 1. GSM instrument at the Dome C site during winter 2006.

sensitive to vibrations since it corresponds to the declination axis) is computed, giving access to the outer scale $\mathcal{L}_{0}$ through a model fitting. Indeed, these covariances are normalized by the differential AA variance obtained with each telescope and are then compared to a grid of theoretical covariances calculated for different baseline coordinates and different $\mathcal{L}_{0}$ values in the case of von Kàrmàn model (Ziad et al. 2000). Theoretical covariances are interpolated for the specific value of the baseline (actually, a projection of the baseline on the wavefront plane is computed first for the moment of observation from the site and source coordinates) and the appropriate $\mathcal{L}_{0}$ that fits the measured covariances is found. The final adopted value of $\mathcal{L}_{0}$ is taken as the median of the 6 individual values corresponding to the different baselines. Figure 2 shows two series of these normalized covariances measured by GSM at different baselines (Fig. 4) and fitted with theoretical curves in the case of the von Kàrmàn model. The measurements were obtained on the star gamma Centauri during the transit leading to longitudinal covariances with negative values for intermediate baselines. The results shown in Fig. 2 correspond to extreme cases of obtained measurements. Indeed, the normalized covariances obtained at all baselines are more or less well fitted by the von Kàrmàn model with the median measured outer scale (top panel of Fig. 2). Another case is shown in the bottom panel of Fig. 2 where the measured covariances are dispersed and only some of them are well fitted by the theoretical curve with the median measured outer scale. This example highlights the importance of the choice of the median value of the 6 baselines measurements.

The configuration of the Dome C GSM version is shown in Fig. 3. The distance between the telescopes is $1 \mathrm{~m}$ in the NorthSouth direction when the observed star transits at the meridian (left panel of Fig. 3). Before and after the star transit, the subapertures of the 2 telescopes are not aligned and the baselines depend on the star position. A projection of these ground baselines on the wavefront plane is performed as described by Avila et al. (1997). The AA measurement in the $X$-direction is aligned with the declination $\delta$ and with right ascension $\alpha$ in the $Y$-direction. As the star diurnal movement compensation by the mounts is in $\alpha$, we decided to avoid the $Y$-direction AA measurement which is contaminated by the vibrations due to the mount driving.

The telescope vibration problem was solved by protecting the instrument with wind screens (Fig. 4) and adding counterweights on the telescope mounts. Vibrations were reduced to an acceptable value as long as the wind speed is lower than 3 to $4 \mathrm{~m} / \mathrm{s}$, which occurs $80 \%$ to $90 \%$ of the time (Valenziano et al. 1999). Different tests were performed to find
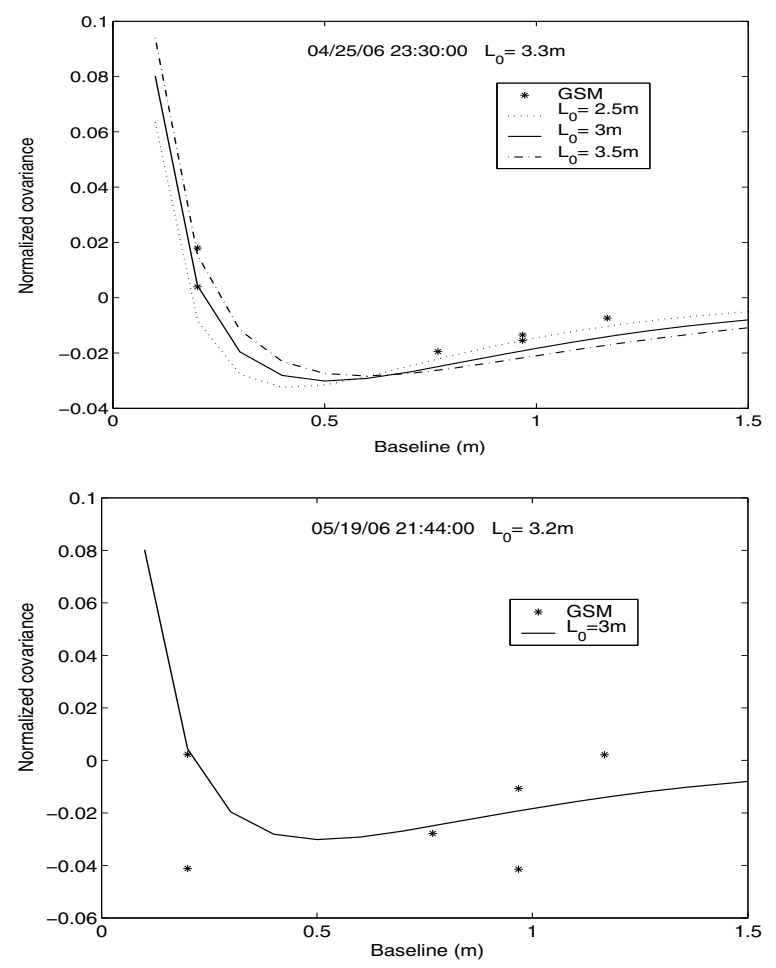

Fig. 2. Examples of normalized covariances measured at different baselines with GSM and fitted with theoretical curves in the case of von Kàrmàn model for outer scale values around the median measured one.
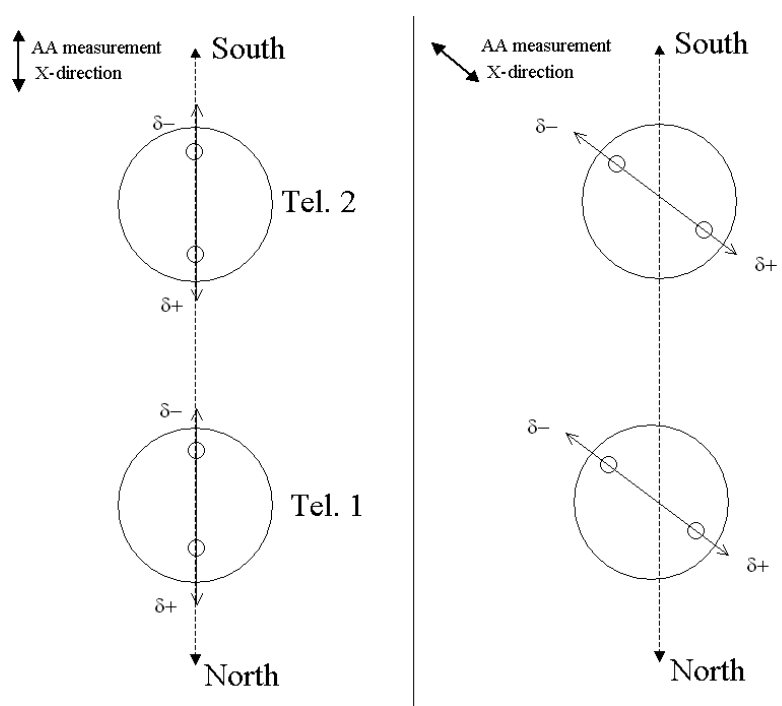

Fig. 3. GSM configuration for AA measurement at the Dome C site.

the best configuration for the orientation of the 2-hole mask of each telescope.

Despite these preventive measures to avoid telescope vibrations, the AA fluctuations could maintain some residual effects, particularly due to the wind. These telescope residual vibrations increase the measured covariances leading to large outer scale values. Thus, the outer scales greater than $1 \mathrm{~km}$ are unreliable and are systematically rejected, especially when appearing only on some of the 6 individual baselines. These large $\mathcal{L}_{0}$ values represent $\sim 20 \%$ of the 33876 total measured outer scales at all baselines for 27 days during the Dome $C$ winter of 2006. Generally, these large $\mathcal{L}_{0}$ are rejected since the adopted outer scale is the 


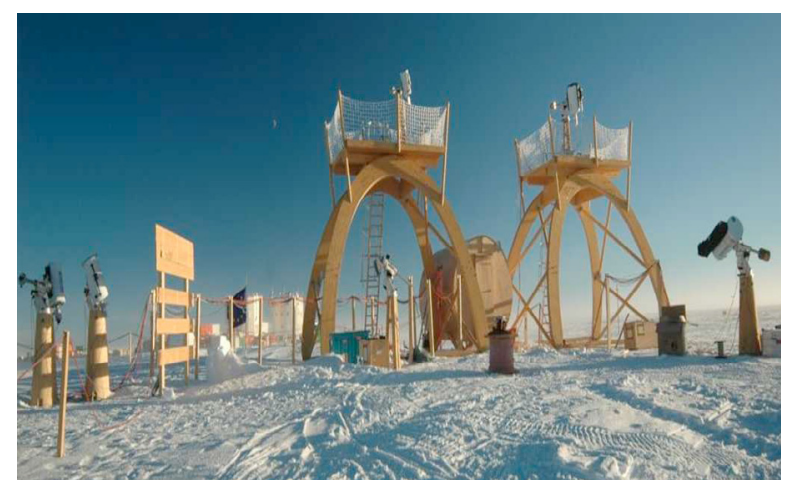

Fig. 4. Site-testing instrumentation at Dome C during winter 2006. The GSM instrument (left) is protected from the prevailing wind by a wood screen.

median of the 6 baseline values. This median filter is very efficient since only $0.4 \%$ and $1.4 \%$ of the 5646 median measured $\mathcal{L}_{0}$ are larger than $100 \mathrm{~m}$ and $50 \mathrm{~m}$ respectively which could be suspected to still be contaminated by vibrations. For this case, a comparison between the absolute seeing measured with individual subapertures $\varepsilon_{0, i}(i=1$ to 4$)$ and the differential seeing $\varepsilon_{0}$ measured by the DIMM method (only in the $X$-direction) has been performed. The difference between the $\varepsilon_{0, i}$ and the $\varepsilon_{0}$ gives information regarding the vibrations existence but this is not sufficient if these vibrations are uncorrelated between the two telescopes. This correlation between the vibrations is visible when the $\varepsilon_{0, i}$ are particularly close between the two telescopes. For the major large outer scale values (larger than $100 \mathrm{~m}$ ) measured at Dome $\mathrm{C}$, we have noticed that when the relative difference between $\varepsilon_{0, i}$ and $\varepsilon_{0}$ is greater than $10 \%$ the $\varepsilon_{0, i}$ are different by at least $20 \%$. We consider, therefore, that the corresponding $\mathcal{L}_{0}$ are not contaminated by vibration. Even if so, this should not affect the statistics since, as indicated above, the percentage of the large outer scales measured at Dome $\mathrm{C}$ is very low.

For some positions of the star, it happens that the two telescopes bump into each other. This problem was resolved for the 2007 winter season, but it caused a lot of down time in 2006 GSM observations. We took advantage of this down time to perform isoplanatic angle estimations, which requires only one telescope.

Estimation of the isoplanatic angle can be made through scintillation measurement of a single star as described by Ziad et al. (2000). Best results are obtained when the input pupil of the telescope has a $10 \mathrm{~cm}$ diameter with a central obstruction of $4 \mathrm{~cm}$ diameter. A dedicated mask was built for that purpose, to be used in front of one of the $\mathrm{C} 11$ telescopes. We used one of the GSM modules to monitor the isoplanatic angle during the time when the outer scale measurements could not be performed.

\section{Data and results}

During the beginning of the 2006 winter-over at Concordia, the GSM was tested and prepared for outer scale measurement simultaneously with seeing using the DIMM method. The data processing was also tested in real conditions to provide outer scale estimations in quasi real-time. The first measurements of outer scale were obtained at the beginning of March 2006. GSM was operational for about 3 months, until the end of May, when the telescope motors failed. Statistics of the outer scale during these 3 months collected by one of us (E.A.) are presented in Figs. 5 and 6. During this local autumn season, there is no
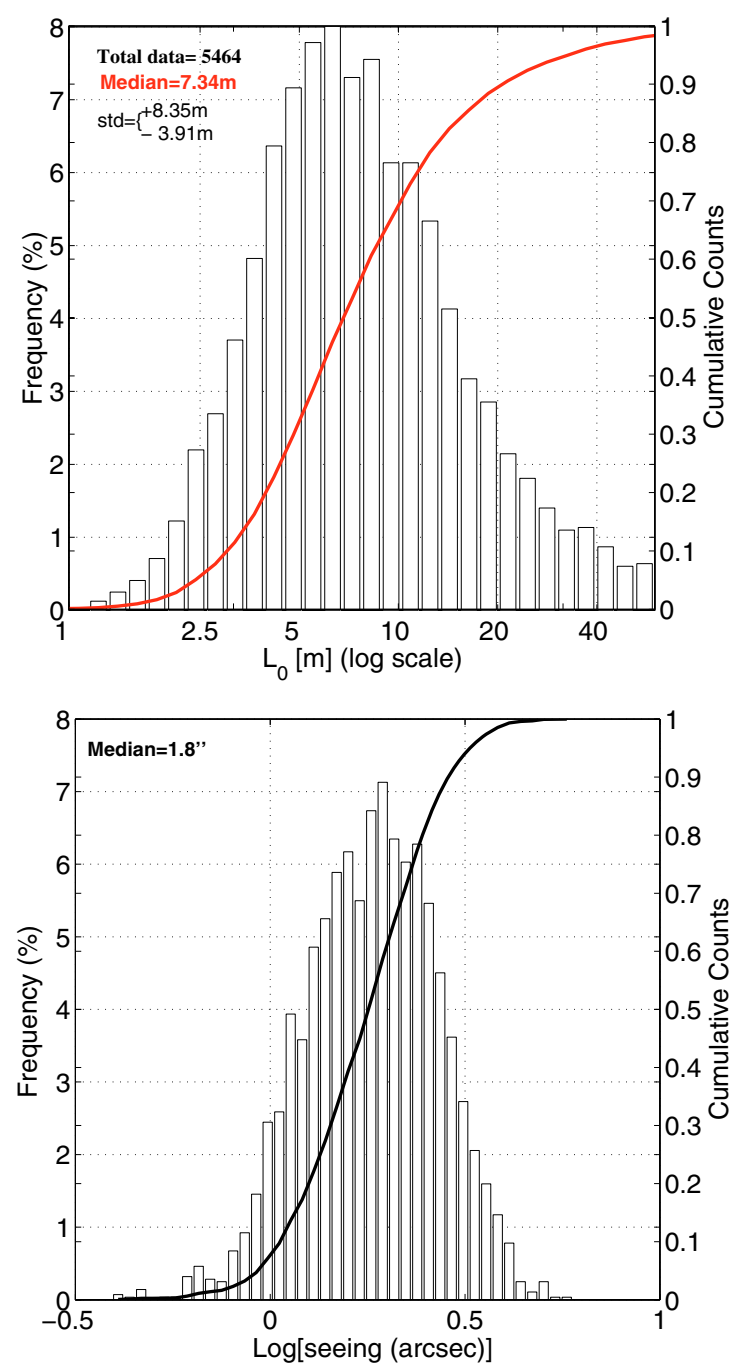

Fig. 5. Outer scale $\mathcal{L}_{0}$ and seeing histograms and cumilative distributions obtained at Dome $\mathrm{C}$ during 3 month measurements from March to May 2006. The seeing is given at $\lambda=500 \mathrm{~nm}$.

permanent night and the measurement ran even in the daytime. Various minor technical problems and bad weather resulted in a total number of measured outer scales at all 6 baselines of 33876 providing 5646 median $\mathcal{L}_{0}$. Figure 5 shows the global histograms and the cumulative distributions of the outer scale and seeing. One can see that the outer scale median value is smaller than in other major sites (Ziad et al. 2000). Indeed, with a $\sim 7.5 \mathrm{~m}$ median outer scale, Dome $\mathrm{C}$ presents an outer scale of $\sim 3$ times lower than Paranal and $\sim 2$ times lower than Mauna Kea. As indicated in Sect. 2, the outer scale measured by the GSM instrument is model-dependent (von Kàrmàn model) and the use of other models should modify the $\mathcal{L}_{0}$ results (Ziad et al. 2000). These low values of $\mathcal{L}_{0}$ are beneficial for future large telescopes and long baseline interferometers to be installed on this site. As shown in Fig. 5 the outer scale has a log-normal distribution and $67 \%$ of the $\mathcal{L}_{0}$ values are included in the interval from $3.4 \mathrm{~m}$ to $15.7 \mathrm{~m}$. Figure 5 shows the seeing measured simultaneously by GSM at $3.5 \mathrm{~m}$ above the ground during the same period. The median seeing $\varepsilon_{0, \mathrm{GSM}}$ value is of $1.80^{\prime \prime}$ with standard deviation of $0.75^{\prime \prime}$. As expected, no correlation exists between the outer scale and the seeing measured simultaneously by GSM (Ziad et al. 2000). 


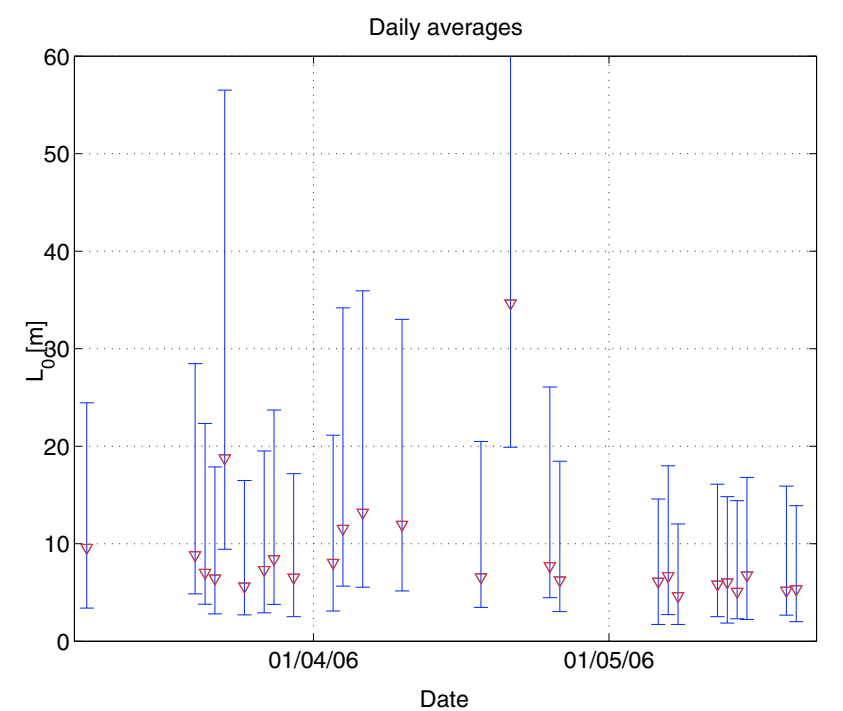

Fig. 6. Outer scale $\mathcal{L}_{0}$ daily variation obtained at Dome $\mathrm{C}$ during 3 month measurements from March to May 2006. The error-bars correspond to the $16 \%$ and $84 \%$ quantiles of the cumulative distribution for each day.

Figure 6 shows daily outer scale measurements. Each day is represented by the $\mathcal{L}_{0}$ median value and the error bars correspond to the $16 \%$ and $84 \%$ quantiles of the cumulative distribution. The gaps in the data are generally due to bad weather conditions and rarely to mechanical problems of mount driving. For some days $\mathcal{L}_{0}$ presents large value and a wide dispersion that could be due to residual vibrations.

Figure 7 shows the diurnal evolution of the outer scale. The gap in Fig. 7 data is the down-time due to the incompatibility between the 2 GSM telescopes (Sect. 2). A decrease in $\mathcal{L}_{0}$ value from $2 \mathrm{pm}$ local time is seen. This behaviour could be explained by the surface layer (SL) evolution. This SL dominated in terms of seeing and turbulence energy (Agabi et al. 2006). At Dome C, a DIMM is installed on a platform at $8.5 \mathrm{~m}$ above the ground (Fig. 4) running permanently and providing a seeing $\varepsilon_{0, \mathrm{DIMM}} \mathrm{ev-}$ ery $2 \mathrm{mn}$. For the same period from March to May 2006, the median seeing $\varepsilon_{0, \text { DIMM }}$ measured by DIMM is $0.88^{\prime \prime}$ when the median seeing $\varepsilon_{0, \mathrm{GSM}}$ obtained by GSM installed at $3.5 \mathrm{~m}$ is, as indicated above, almost 2 times larger. This is, once again, a strong indication of the domination of the surface layer at Dome C (Agabi et al. 2006). Thus, the outer scale measured by GSM refers generally to this surface layer. A direct consequence is an anisotropy of the outer scale due to the behaviour of this prevailing surface layer. It is evident that if a telescope is installed higher than the Dome $\mathrm{C}$ surface layer, it would be sensitive to an outer scale corresponding to the free atmosphere.

During the outer scale measurements down time, as explained above (Sect. 2) we used one of the GSM modules to monitor the isoplanatic angle $\theta_{0}$. The $\theta_{0}$ measurements and statistics were collected during 4 months from February to May 2006 until the breakdown of the telescope motor. The results are shown in Fig. 8 at $\lambda=500 \mathrm{~nm}$. They are comparable to what was obtained in 2005 with the same equipment (Agabi et al. 2006).

\section{Discussion and conclusion}

For the first time, we have statistics on the wavefront outer scale at the Dome $\mathrm{C}$ site in Antarctica. The $\mathcal{L}_{0}$ has a log-normal distribution with a quite small median value compared to major

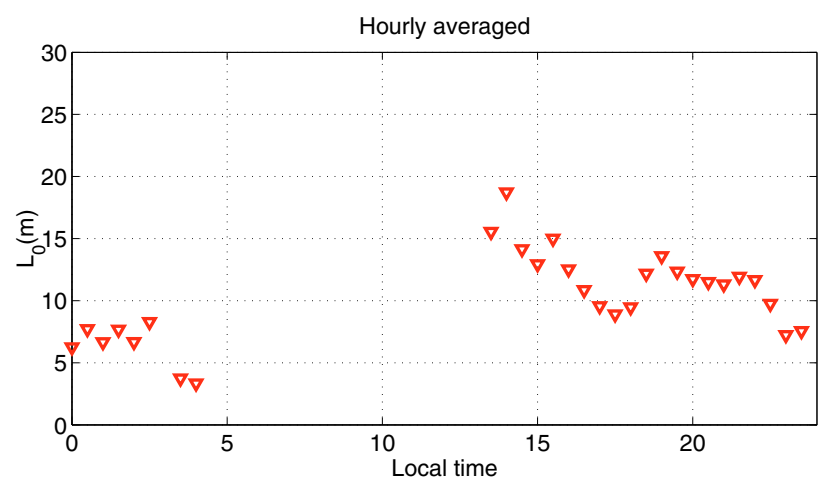

Fig. 7. Outer scale $\mathcal{L}_{0}$ diurnal variation obtained at Dome $\mathrm{C}$ during 3 month measurements from March to May 2006.

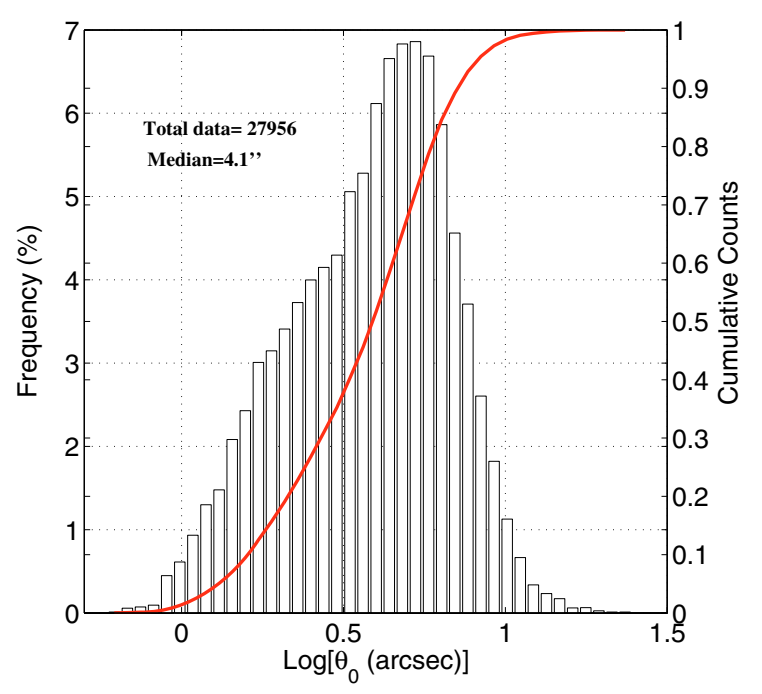

Fig. 8. Isoplanatic angle histogram and cumulative distribution obtained during the period February-May 2006 at $\lambda=500 \mathrm{~nm}$.

sites around the world (Ziad et al. 2000). The Dome C outer scale values are 2 to 3 times lower than Mauna Kea and Paranal Observatories respectively. Due to a prevailing surface layer (SL) at Dome C, the measured outer scale refers to this SL which is known to have an anisotropic behaviour. These small $\mathcal{L}_{0}$ values will lead to better performances of the HAR instruments which confirms, once again, the usefulness of the Dome $\mathrm{C}$ site. The outer scale has a strong impact on HAR techniques such as AO systems and interferometry. Indeed, design, performance and optimization of adaptive optic systems are related to atmospheric considerations and notably to the wavefront outer scale amplitude. Several analyses of outer scale effects in terms of Zernike decomposition of the atmospherically induced phase aberrations were performed theoretically (Winker 1991; Sasiela \& Shelton 1993; Ellerbroek 1997; Voitsekhovich \& Cuevas 1995). Ragazzoni et al. (2005) noted that tip-tilt aberration for an ELT could be corrected with the same mirror used to correct higher order modes.

The variances of the amplitude of atmospheric Zernike coefficients found using the Dome $\mathrm{C}$ median outer scale exhibit differences for the tip-tilt modes but no significant difference for higher order modes. Figure 9 represents the variances of the amplitude of atmospheric Zernike coefficients calculated with the formalism described by Winker (1991). We use the median outer scale $\mathcal{L}_{0}$ measured at Dome $\mathrm{C}$ to calculate these variances for different telescope sizes. One can notice that for telescopes 


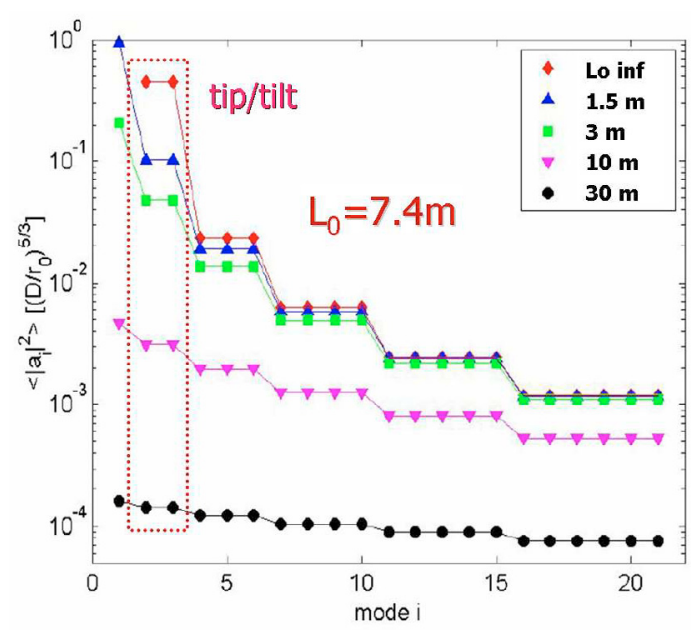

Fig. 9. Normalized variances of atmospheric Zernike coefficients for different telescope diameters and the Dome $\mathrm{C}$ median outer scale $\mathcal{L}_{0}$ obtained during 3 month measurements from March to May 2006. The case $\mathcal{L}_{0}=\infty$ (diamond) corresponds to the Kolmogorov model.

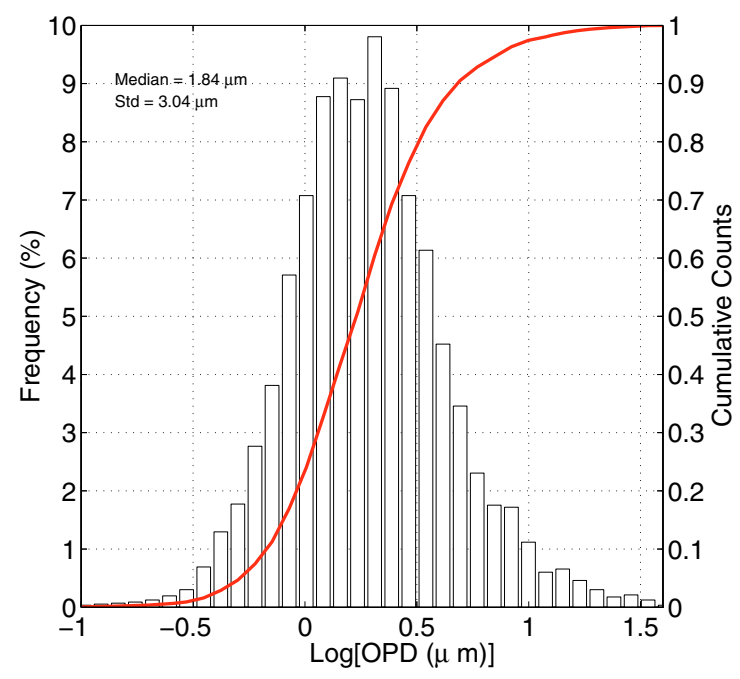

Fig. 10. Fringe excursion histogram obtained for an interferometer of $100 \mathrm{~m}$ baseline and from the Dome $\mathrm{C}$ outer scale $\mathcal{L}_{0}$ and seeing statistics obtained during 3 month measurements from March to May 2006.

larger than $10 \mathrm{~m}$, the tip-tilt contribution is more strongly reduced than the other modes, leading to less motion of the images in the telescope focal plane. For a $30 \mathrm{~m}$ ELT, the tip-tilt should be corrected by the AO system without need for a separate tip-tilt correction.

In interferometry the fringe excursion is strongly dependent on the outer scale according to the telescope diameter and the baseline (Mariotti 1994; Conan et al. 2000; Ziad et al. 2004; Maire et al. 2006). As the measured outer scale at Dome $C$ is smaller than at mid-latitude observatories, it is, then, expected that the fringe excursion should be smaller. As shown in Fig. 10, the standard deviation of the optical path difference $\sigma_{\text {OPD }}$ (Maire et al. 2006) for an interferometer at Dome $\mathrm{C}$ has a log-normal

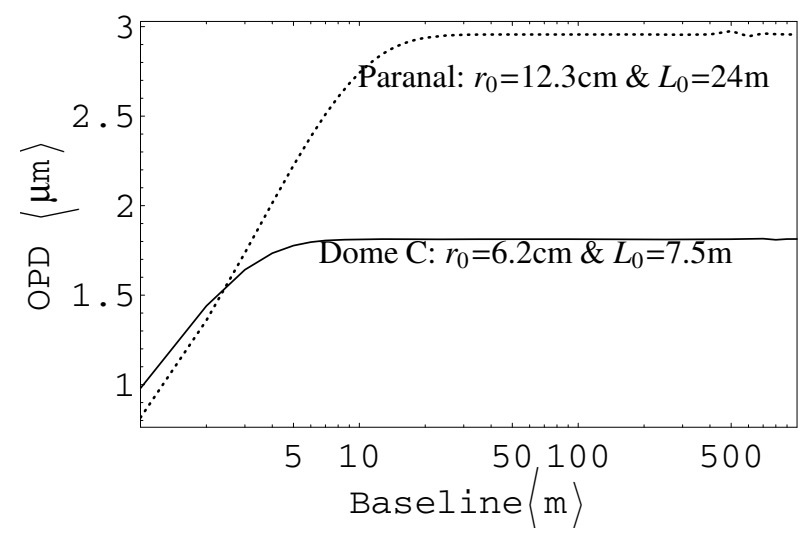

Fig. 11. OPD versus baseline for an interferometer of $1 \mathrm{~m}$ telescope diameter in the case of Dome C (full line) and Paranal (Dashed line). Only the median values of seeing and outer scale are used for both sites.

distribution with a median value of $1.84 \mu \mathrm{m}$ for a $1 \mathrm{~m}$ telescope diameter and $100 \mathrm{~m}$ baseline. For the same interferometer at Paranal the $\sigma_{\mathrm{OPD}}$ is $\sim 3 \mu \mathrm{m}$ for median values of seeing and outer scale measured with the GSM (Ziad et al. 2000). The OPD at Dome $\mathrm{C}$ is $\sim 2$ times smaller than at Paranal even if the seeing was poor due to a strong surface layer (Agabi et al. 2006). This result shows that the outer scale has more impact on the fringe excursion than the seeing. Indeed, as shown in Fig. $11 \sigma_{\text {OPD }}$ is more sensitive to the seeing $\left(1.8^{\prime \prime}\right.$ and $0.91^{\prime \prime}$ respectively for Dome $\mathrm{C}$ and Paranal) for baselines shorter than the outer scale (7.5 $\mathrm{m}$ and $24 \mathrm{~m}$ respectively for Dome $\mathrm{C}$ and Paranal) but for longer baselines the outer scale dominates.

Thus, these small outer scales measured at Dome C combined with a long coherence time (Agabi et al. 2006) and with a large isoplanatic angle should make it easier to optimise the HAR techniques in use at this site.

Acknowledgements. We wish to thank the summer camp logistics and the winterover team at Concordia for their help. The program is supported by the Polar Institutes IPEV and PNRA.

\section{References}

Avila, R., Ziad, A., Borgnino, J., et al. 1997, J. Opt. Soc. Am. A, 14, 3070 Agabi, A., Aristidi, E., Azouit, M., et al. 2006, PASP, 118, 344 Aristidi, E., Agabi, A., Fossat, E., et al. 2005, A\&A, 444, 651 Borgnino, J., Martin, F., \& Ziad, A. 1992, Opt. Comm., 91, 267

Conan, R., Ziad, A., Borgnino, J., Martin, F., \& Tokovinin, A. 2000, Proc. SPIE, 4006, 963

Ellerbroek, B. L. 1997, Appl. Opt., 36, 9456

Maire, J., Ziad, A., Borgnino, J., et al. 2006, A\&A, 448, 1225

Mariotti, J.-M. 1994, Adaptive optics for long baseline interferometry, Adaptive Optics for Astronomy, ed. D.-M. Alloin, \& J.-M. Mariotti (Kluwer Academic Publisher), 309

Ragazzoni, R., Le Roux, B., \& Arcidiacono, C. 2005, C. R. Physique, 6, 1081

Sasiela, R. J., \& Shelton, J. D. 1993, J. Opt. Soc. Am. A, 10, 646

Valenziano, L., \& dall'Oglio, G. 1999, PASA, 16, 167

Voitsekhovich, V. V., \& Cuevas, S. 1995, J. Opt. Soc. Am. A, 12, 2523

Winker, D. M. 1991, J. Opt. Soc. Am. A, 8, 1568

Ziad, A., Conan, R., Tokovinin, A., Martin, F., \& Borgnino, J. 2000, Appl. Opt., 39,5415

Ziad, A., Schöck, M., Chanan, G. A., et al. 2004, Appl. Opt., 43, 2316 